\title{
Towards a general record linkage framework for statistical disclosure control *
}

\author{
Duncan Smith \\ University of Manchester \\ Oxford Road \\ Manchester, England \\ duncan.g.smith@manchester.ac.uk
}

\author{
Mark Elliot \\ University of Manchester \\ Oxford Road \\ Manchester, England \\ mark.elliot@manchester.ac.uk
}

\begin{abstract}
The assessment of statistical disclosure risk often requires the linking of data. There are effective means of linking data for simple scenarios; but it is not clear how best to approach linkage for more complex scenarios. We examine linkage approaches for three simple scenarios and argue that they might be combined.
\end{abstract}

\section{CCS Concepts}

- Mathematics of computing $\rightarrow$ Probabilistic inference problems; Bayesian computation;

\section{Keywords}

Statistical disclosure; record linkage; Bayes

\section{INTRODUCTION}

Statistical disclosure control concerns the security of data in a statistical sense. Can the data be released without compromising the privacy of the data subjects? Data that are deemed to be secure can be released to the intended end-users. Data that are not considered secure might be either perturbed or partially suppressed to make them secure before being released.

The starting point for risk assessment is often to define an attack scenario [?]. This describes the data intruder (person or organization that might launch an attack), the relevant information assumed to be held by the data intruder, and the means by which the data intruder would attack the data (if released). Given the attack scenario we can assess risk by putting ourselves in the position of the data intruder and attacking the data. The attack often incorporates some form of record linkage.

We define a record as data relating to a single entity so we depart from the usual definition of a vector of values

\footnotetext{
* This research was supported by the National Centre for Research Methods with funding from the ESRC: ES/L008351/1
}

Permission to make digital or hard copies of part or all of this work for personal or classroom use is granted without fee provided that copies are not made or distributed for profit or commercial advantage and that copies bear this notice and the full citation on the first page. Copyrights for third-party components of this work must be honored. PrAISe '16 August 29-30, 2016, The Hague, Netherlands

(C) 2016 Copyright held by the owner/author(s).

ACM ISBN 978-1-4503-4304-6/16/08.

DOI: http://dx.doi.org/10.1145/2970030.2970037

\section{(c) (i)}

This work is licensed under a Creative Commons Attribution International 4.0 License. for a corresponding vector of variables. We do not generally assume that the data are error free, or that the record contains data that can directly identify the entity. A record can contain arbitrary data, including relationships to (the subjects of) other records.

Related entities might be of different types. Assume that the entity that is the subject of a record is a person. Then we might have data regarding familial or social network relationships with other persons; but we might also have ownership relationships relating the entity to credit cards or pets.

Record linkage is the connecting of records via relationships. In many applications the relationship is identity records are linked if they are considered to relate to the same entity. In this case linked records can be merged to create a single record. Here we entertain the possibility of alternative link / relationship types such as those mentioned above.

Statistical disclosure arises when linkage allows an entity to be both identified and associated with sensitive information, and with a sufficiently high degree of confidence. This is a very general definition, but the important features are that the data intruder can identify the entity, and that the data intruder learns something new about the entity.

In order to assess disclosure risk we need to be able to consider how a data intruder might use all available data to launch an attack. If we consider an attempt to link a pair of records there are perhaps three main categories of data that we need to consider.

1. Observed variables that are common to both records

2. Observed variables that appear in only one record

3. Observed links between the (subjects of the) records

\section{LINKING ON COMMON VARIABLES}

Fellegi and Sunter [?] address the identity linkage problem for two datasets $A$ and $B$. The underlying assumption is that $A$ and $B$ are random samples from a common population.

There is a set of all possible matches.

$$
A \times B=\{(a, b) ; a \in A, b \in B\}
$$

This can be partitioned into sets of linked and unlinked pairs,

$$
\begin{gathered}
M=\{(a, b) ; a=b, a \in A, b \in B\} \\
U=\{(a, b) ; a \neq b, a \in A, b \in B\}
\end{gathered}
$$


and we wish to allocate the possible matches to these sets.

Fellegi and Sunter only exploit the data in variables that are common to $A$ and $B$. Thus the records in $A$ and $B$ can be aligned so that these variables are indexed from 1 to $n$. e.g. $a=\left\{a_{1}, \ldots, a_{n}\right\}$.

Fellegi and Sunter also adopt the assumption that the matching variables are conditionally independent given the true link status. They compare values solely in terms of equality. Thus we have the following expression for the posterior odds of a link,

$$
\begin{array}{r}
\frac{\operatorname{Pr}((a, b) \in M \mid(a, b))}{\operatorname{Pr}((a, b) \in U \mid(a, b))}=\left(\prod \frac{\operatorname{Pr}\left(\left(a_{i}=b_{i}\right) \mid(a, b) \in M\right)}{\operatorname{Pr}\left(\left(a_{i}=b_{i}\right) \mid(a, b) \in U\right)}\right) \\
\times \frac{\operatorname{Pr}((a, b) \in M)}{\operatorname{Pr}((a, b) \in U)} .
\end{array}
$$

The numerator and denominator terms in the Bayes factor are termed $m$ and $u$ probabilities respectively. It is not generally assumed that the $m$ probabilities are equal to 1 . Thus the approach can accommodate typographical (and other) errors. Jaro [?] presents an Expectation Maximization (EM) [?] algorithm for generating maximum likelihood estimates for all the required parameters (the $m$ and $u$ probabilities and the marginal probability of a link, $\operatorname{Pr}((a, b) \in M)=1-\operatorname{Pr}((a, b) \in U)$. Fellegi and Sunter [?] present a decision rule for allocating record pairs to $M$, $U$ or a third category of uncertain matches. The uncertain matches are subjected to clerical review - the record pairs are examined by a human who manually allocates the pair to either $M$ or $U$. The reviewer will generally consider the complete records, rather than solely the common variables.

Many improvements to the basic scheme have been proposed over the years. Some are reviewed and discussed in [?]. Fellegi-Sunter linkage requires no prior information or additional training data.

\section{LINKING WITHOUT COMMON VARI- ABLES}

Identity linkage without common variables has been addressed by Torra [?] and Nin and Torra [?]. They use OWA (ordered weighted averaging) operators to generate representatives - new variables that are generated from structural information that is common to the variables in $A$ and the variables in $B$.

An OWA operator of dimension $N$ can simply be specified as a vector $W=\left[w_{1}, \ldots, w_{N}\right]$ of non-negative weights that sum to one. The operator computes the weighted mean of an ordered vector,

$$
O W A\left(x_{1}, \ldots, x_{N}\right)=\sum_{j=1}^{N} w_{j} y_{j}
$$

where $y_{j}$ is the $j$ th largest of the $x_{i}$.

Torra [?] specifies an OWA operator via a non-decreasing fuzzy quantifier. This is simply a non-decreasing function $F$ with domain $[0,1]$ and range[0,1]. The weights for an OWA operator of length $N$ are then calculated as,

$$
w_{j}=F(j / N)-F((j-1) / N) .
$$

Thus we have a single specification that can generate OWA operators for vectors of different lengths.
Initially, the variables in $A$ and $B$ are processed via either a translation to the unit interval (range normalisation), or via standardizing so that the variable values have mean 0 and variance 1 . A set of non-decreasing fuzzy quantifiers is applied to the records in $A$ and $B$ to generate a set of new variables. Classical record linkage is applied using the new variables.

Nin and Torra [?] show that the linkage achieved is better than random allocation of links when $A$ contains variables that are highly correlated with variables in $B$. In fact, this is a crucial requirement. If the approach is not limited to highly correlated variables it tends to perform in line with random allocation [?].

Smith [?] compares OWA linkage with a simple Bayesian approach grounded in (essentially) the same basic theory that underpins Fellegi-Sunter linkage. If we have a model $G$ for the joint distribution of the variables in $A$ and $B$, then we can estimate the posterior odds via,

$$
\frac{\operatorname{Pr}((a, b) \in M \mid(a, b))}{\operatorname{Pr}((a, b) \in U \mid(a, b))}=\frac{\operatorname{Pr}(a \oplus b \mid G)}{\operatorname{Pr}(a \mid G) \operatorname{Pr}(b \mid G)} \frac{\operatorname{Pr}((a, b) \in M)}{\operatorname{Pr}((a, b) \in U)}
$$

where $\oplus$ denotes concatenation.

In [?] $G$ is a decomposable graphical model fitted from training data. $\operatorname{Pr}((a, b) \in M)$ is estimated from the Bayes factors using EM. The Bayesian approach is shown to consistently outperform OWA linkage.

Alternative methods such as clustering can also be used $[?, ?]$. These require the specification of similarity measures or distance measures.

The above approaches for linkage without common variables all require some form of prior information or additional training data. OWA linkage requires prior information on correlations. The Bayesian approach requires prior information regarding statistical dependencies. Clustering methods require the specification of appropriate similarity / distance measures.

\section{LINKAGE USING KNOWN RELATION- SHIPS}

Here we are interested in graphs that have nodes corresponding to entities and edges corresponding to e.g. familial, social network or ownership links between entities. Such graphs have been used for identity linkage. Backstrom et al. [?] considered both active and passive attacks on social network graphs. (An active attack involved setting up accounts and social network relationships that would generate readily identifiable graph structures.) Fu et al. [?] combined record linkage and a form of graph linkage in order to match on households.

In principle we want to be able to infer many types of link between entities - not just identity. Furthermore, we want to be able to take into account observed variables and observed links when making inferences about the unobserved variables and unobserved links. Getoor et al. [?] discusses probabilistic relational models (PRMs). These are designed to capture both probabilistic interactions between the variables of related entities and probabilistic interactions between variables and the link structure itself. Taskar et al. [?] present a relational modelling approach based on Markov graphs. They call their models relational Markov networks (RMNs). RMNs are undirected graphs, and are easier to deal with than PRMs, which are directed and must be acyclic. In 
Taskar et al. [?] links are treated as binary variables (exists, does not exist) and data associated with the link endpoint entities is used to generate a posterior belief over the existence of the link.

The relational graphical modelling approach is very attractive. Dependencies between relevant variables are described by a probabilistic graphical model. A link between a pair of entities implies dependencies between the variables in the corresponding models. Thus a link connects a pair of models, producing a new model. For uncertain links the ratio of the data likelihoods under the linked and unlinked models gives us a Bayes factor that can be multiplied by the prior odds (of a link) to give us the posterior odds. We can use model averaging (over linkage structures) to generate posterior beliefs over unobserved variables.

Relational graphical modelling provides a clear mechanism for performing the inferences in which we are interested. We can make inferences about unobserved variables / links based on observed variables / links. Identity linkage is a special case where the endpoint entities are of the same type and are described by the same graphical model. In fact, the implementation in [?] also used a graphical modelling approach. As it concerned identity linkage and there were no common observed variables only a single model (for the union of the variables in $A$ and $B$ ) was required.

\section{DISCUSSION}

There are existing Bayesian approaches that are effective at identity linkage on common variables, and on variables that are not common to both records. There is also the (Bayesian) relational graphical modelling approach for inferring the existence of non-identity links. These approaches are based on the same underlying theory. They all estimate the posterior odds of a link by multiplying prior odds by a Bayes factor. They mainly differ in the treatment of the Bayes factor - for instance, by focussing on equality of values in the Fellegi-Sunter approach. We should, in principle, be able to combine these approaches and produce a general framework for linkage.

Combining the approach of Fellegi and Sunter [?] with that of Smith [?] would be expected to provide better (identity) linkage than Fellegi-Sunter linkage alone. Variables that are not common to both $A$ and $B$ are usually only considered for record pairs that are sent for clerical review. In principle, the approach developed in [?] might provide some kind of automated clerical review. Fellegi-Sunter would do the heavy lifting using the common variables, and the data in the remaining variables would be exploited for only the uncertain links. There is an implicit naive Bayes assumption in applying both methods without adjustment, but for an iterative approach we might get reasonable results. An alternative would be to combine the approaches at a more basic level, removing the aforementioned naive Bayes assumption.

The Fellegi-Sunter approach is based on binary comparisons, because for identity linkage we would generally expect values for the same variable to be equal if a match is correct. If the match is incorrect, then the probability of equality for some variables can be very low. Thus Fellegi-Sunter linkage can be very discriminating. For other types of linkage we cannot generally limit ourselves to such simple comparisons. So an important question is what kinds of comparisons can we make for different types of link? Both [?] and [?] condition upon observed variables. In fact, one factor that makes the combination of the approaches in [?] and [?] non-trivial is this difference between the aspects of the data that are conditioned upon.

Due to the common underlying theory there are possibilities for combining these approaches. Yet there are still limitations. Sadinle and Fienberg [?] demonstrated the difficulty of generalizing the Fellegi-Sunter approach to more than two datasets. Of course, the number of combinations of records increases exponentially with the number of datasets $K$. But for each combination the number of matching patterns is equal to the $K$ th Bell number. Sadinle and Fienberg report good results for $K=3$, but clearly there is an issue of scale. There are also issues of scale with the relational graphical modelling approach, where links can create enormous graphs and generating posterior beliefs becomes computationally costly.

There is also an issue with the data which might be met in practice. The underlying assumption of Fellegi-Sunter linkage is that there are two datasets that are random samples from a common population. Other approaches require training data (that are assumed to have nice statistical properties). Under many attack scenarios these assumptions will not hold. The data intruder might be attempting to link adminstrative data with social network data or data derived from blog / usenet postings. This is clearly a problem if we want good record linkage performance. Of course, this is also a problem for the data intruder. The real issue is that it can make risk assessment more difficult. We cannot be quite as sure that the data intruder is not capable of performing better linkage than we are. Therefore, approaches that can deal with such poor quality data must be developed. Combining the approaches in [?], [?] and [?] is not problematic in terms of theory, but is not trivial in practice. The more difficult issues relate to machine learning algorithms and parameter estimation. Fellegi-Sunter often uses EM, whereas graphical model determination can use a variety of algorithms ([?] uses a Bayesian approach). Learning clearly becomes more problematic when usual assumptions are violated, and more so when there are also issues of scale.

\section{REFERENCES}

[1] L. Backstrom, C. Dwork, and J. Kleinberg. Wherefore art thou r3579x?: Anonymized social networks, hidden patterns, and structural steganography. In Proceedings of the 16th International World Wide Web Conference, pages 181-190, 2007.

[2] S. Bender, R. Brand, and J. Bacher. Re-identifying register data by survey data: An empirical study. Statistical Journal of the United Nations ECE, (18):373-391, 1991.

[3] A. P. Dempster, N. Laird, and D. B. Rubin. Maximum likelihood from incomplete data via the EM algorithm. Journal of the Royal Statistical Society, Series B, 39(1):1-38, 1977.

[4] M. J. Elliot and A. Dale. Scenarios of attack: the data intruder's perspective on statistical disclosure risk. Netherlands Official Stat., (14):6-10, 1999.

[5] I. Fellegi and A. Sunter. A theory for record linkage. JASA, 64(238):1183-1210, 1969.

[6] Z. Fu, P. Christen, and J. Zhou. A graph matching method for historical census household linkage. International Journal of Humanities and Arts Computing, 8:204-225, 2014. 
[7] L. Getoor, N. Friedman, D. Koller, and B. Taskar. Learning probabilistic models of link structure. Journal of Machine Learning Research, (3):679-707, 2002.

[8] M. Jaro. Advances in record linkage methodology as applied to the 1985 census of Tampa Florida. JASA, 84(406):414-420, 1989.

[9] J. Nin and V. Torra. Towards the use of OWA operators for record linkage. In Proceedings of the Joint 4th Conference of the European Society for Fuzzy Logic and Technology and the 11th Rencontres Francophones sur la Logique Floue et ses Applications, Barcelona, Spain, September 7-9, pages 34-39, 2005.

[10] J. Novak, P. Raghavan, and A. Tomkins. Anti-aliasing on the web. In Proc. WWW2004, May 17-22, New York, pages 30-39, 2004.

[11] M. Sadinle and S. E. Fienberg. A generalized Fellegi-Sunter framework for multiple record linkage with application to homicide record systems. JASA, 108(502):385-397, 2013.

[12] D. Smith. Re-identification in the absence of common matching variables. Technical report, University of Manchester. http://hummedia.manchester.ac.uk/ institutes/cmist/archive-publications/working-papers/ 2016/2016-02.pdf.

[13] B. Taskar, M. Wong, P. Abdeel, and D. Koller. Link prediction in relational data. In Advances in Neural Information Processing Systems 16, pages 659-666, 2004.

[14] V. Torra. OWA operators in data modeling and reidentification. IEEE Transactions on Fuzzy Systems, 12(5):652-660, 2004.

[15] W. E. Winkler. The state of record linkage and current research problems. Technical report, U.S. Bureau of the Census. http://www.census.gov/srd/papers/pdf/rr99-04.pdf. 